\title{
Síndrome de feminización total
}

E] síndrome de testículo feminizante o feminización total es una variedad de pseudohermafroditismo masculino con fenotipo femenino. Se trata de individuos portadores de testículos con genitales externos de tipo femenino en la mayoría de los casos, con una vagina que termina en forma ciega y generalmente ausencia de útero y anexos. Los testículos se encuentran localizados ya sea en posición intraabdominal, inguinal o en los labios mayores.

Tanto la orientación psicológica como el aspecto somático son femeninos, llamando, eso sí, la atención una talla generalmente elevada con proporciones eunucoides en las que destacan pies y manos grandes.

El desarrollo puberal es incompleto, presentan desarrollo mamario, pero con aréolas poco pigmentadas y pezones pequeños $y$, en general, ausencia o muy escaso vello sexual, unido siempre a la existencia de una amenorrea primaria. $^{1-2}$

\section{MATERIAL Y METODO}

Nuestra casuística está constituida por una familia cuyo árbol genealógico es analizado a través de tres generaciones, lográndose pesquisar cuatro miembros portadores del síndrome (Fig. 1).

La investigación se realiza en cuatro pacientes a las que se le practican los siguientes estudios:

"Depto, de Endocrinología, Sede Occidente, Hospital San Juan de Dios.

*Depto. de Ginecalogia, Sede Occidente, Hospital San Juan de Dios.

** Depto. de Genética, Sede Occidente, Hospital San Juan de Dios.
Dra. Nelly Abodovsky*, Ximna Vicanco*, Carlos Crisosto **, Isabel Avendaño***, señora Francisca Perez ***

1. Anamnesis. Examen físico y ginecológico.

2. Determinación de cromatina nuclear en frotis bucal.

3. Colpocitograma. Tinción May Grünwald Giemsa.

4. GT urinarias. Método de Klinefelter.

5. 17 KS urinarias. Método del M.R.C., Comitte of Endoc.

6. Captación de yodo 131.

7. Edad ósea.

8. Dermatoglifos.

9. Cariograma.

\section{CASO No 1}

M.C., niña de 14 años de edad, fue la primera en consultar del grupo familiar. La molestia principal es falta de menarquia en presencia de signos puberales de dos ańos de evolución.

Entre sus antecedentes refiere haber sido operada de hernia inguinal bilateral alrededor de los 3 años. A los 12 años relata aparición de caracteres sexuales secundarios, con desarrollo mamario y vello perigenital escaso.

En su familia existen dos tías y una prima, casadas, que presentan amenorrea y esterilidad.

Al examen se encuentra una niña de $165 \mathrm{~cm}$ de estatura con proporciones eunucoides (pubis-suelo 88 , evergadura 178). Llama la atención el gran tamaño de manos y pies. La piel es de caracteres normales y hay ausencia de vello axilar y escasísimo vello perigenital, sin velto pubiano. Existe un aumento de volumen del tiroides, correspondiente a un bocio grado II. En el abdomen hay ci- atrices de herniorrafia inguinal bilateral. Los genitales externos son de aspecto femenino normal. Ex. ginecológico: clítoris pequeño, vagina corta, de 3 a $4 \mathrm{~cm}$ de largo, termina en forma ciega. No se palpa útero ni anexos.

\section{Exámenes de Laboratorio}

Cromatina nuclear: ( - ). Colpocitograma: efecto estrogénico normal. GT urinarios: $11 \mathrm{U}$ st. Captación 1-131: 20\%. Edad ósea: 15 años. Dermatoglifos: papilas altas, gruesas. Conteo total: 96 . Angulo a t d: $44^{\circ}$. Cariograma: 46 $\mathrm{XY}$. 


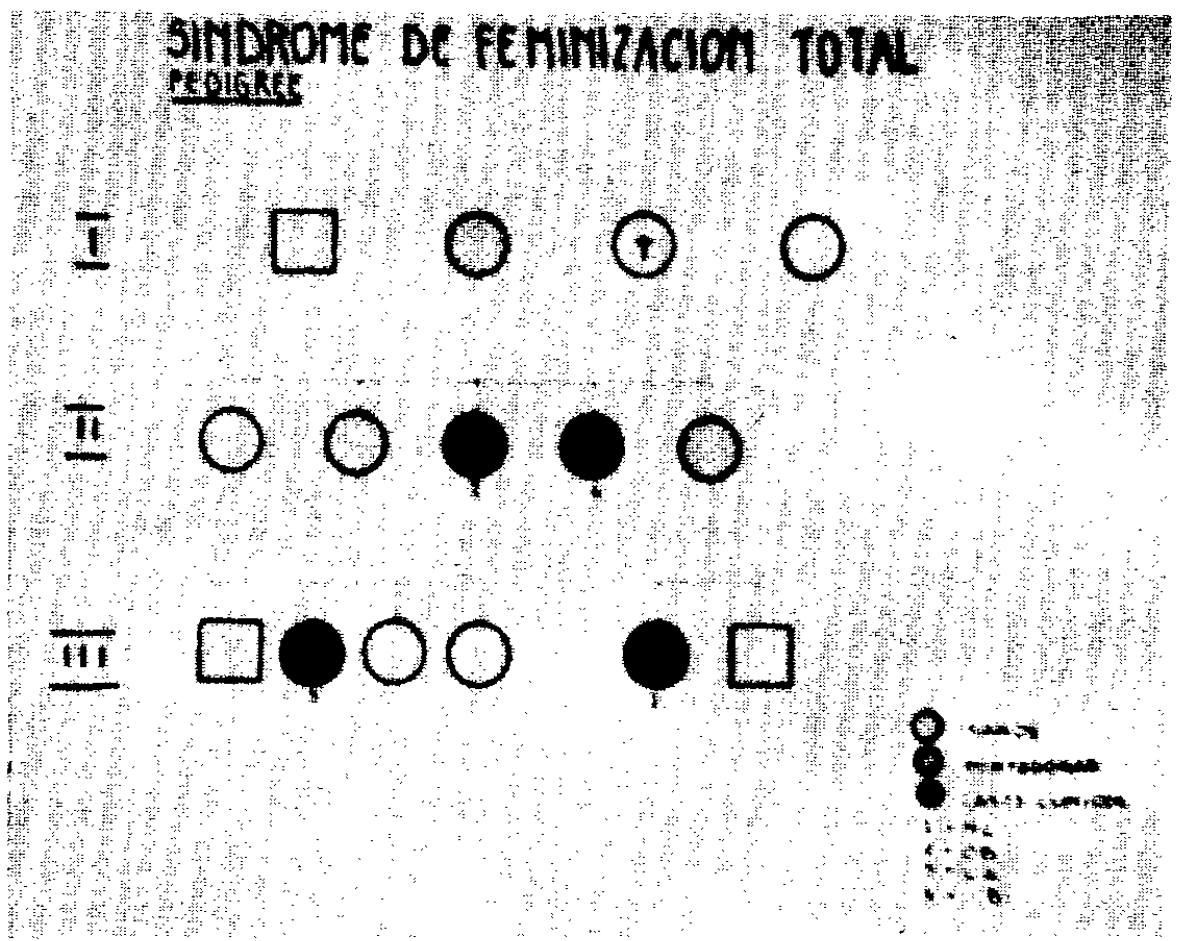

Fig. 1

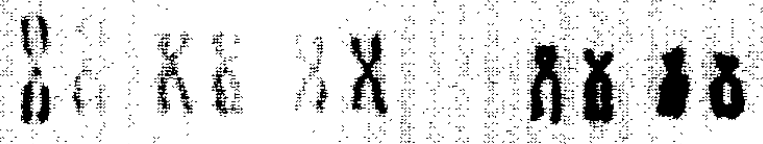

\section{8x $x \times \times 8 \times x \times x \times x n 8$}

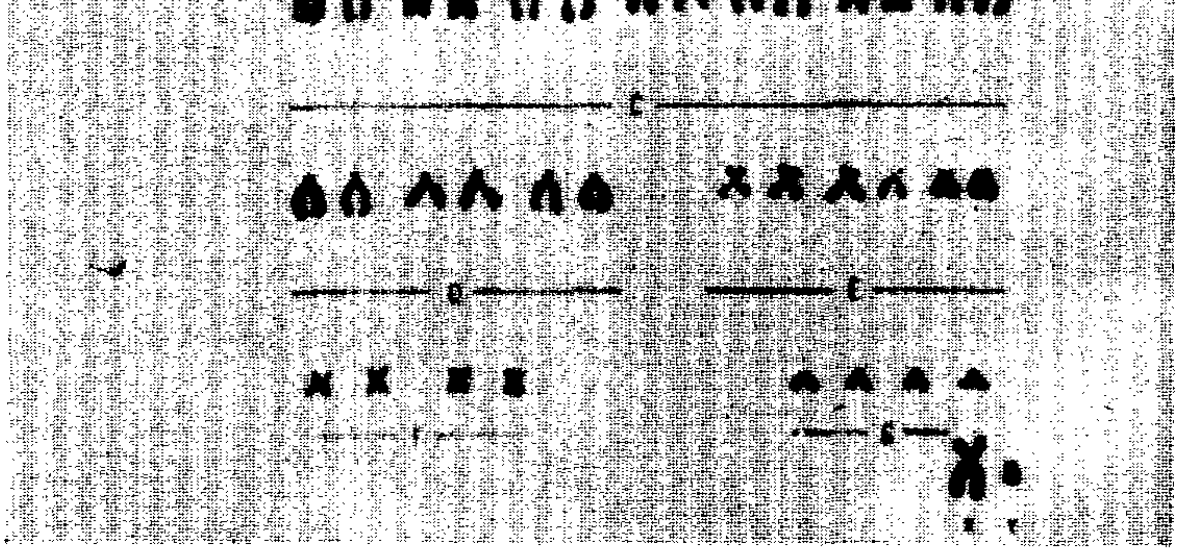

Fig. 2 
O.B., paciente de 37 años, tía de la anterior, casada; sus molestias principales son amenorrea primaria y esterilidad. Relata aparición de desarrollo mamario a los 12 años de edad. Actualmente sus relaciones sexuales son normales, existiendo libido y orgasmo.

Al examen físico: estatura, $160,5 \mathrm{~cm}$. Proporciones eunucoides (pubis-suclo 84, envergadura 165). Obesa, piel de caracteres normales, vello axilar ausente, vello perigenital escaso, sin vello pubiano. Mamas bien desarrolladas, pero con aréolas poco pigmentadas y pezones pequerios. Genitales externos femeninos, normales; clítoris, pequeño; mucosa del introito, normal. Examen gtnecoligico: vagina larga, con mucosa rosada y cutrófica No se visualiza cuello, no se palpa cuerpo ni anexos.

Exámenes de Laboratorio

Cromatina nuclear, (-). Colpocitograma: deficiencia estrogénica moderada. GT urinaria: $10 \mathrm{U}$ st. Dermatoglifos: conteo, 110; ángulo a $1 \mathrm{~d}, 47$. Papilas gruesas. Cariograma: $46 \mathrm{XY}$.

\section{$\mathrm{CASO}^{+} \mathrm{N}^{\circ} 3$}

C.A., 24 años, casada, prima hermana de la primera paciente. Molestias principales: amenorrea primaria y esterilidad. Reliere que entre los 10 y 11 años inicia el desarrollo mamario. A los 13 años es operada de hernia inguinal bilateral. Casada hace 3 años, tiene relaciones sexuales normales, con libido y orgasmo.

Al examen: talla $167 \mathrm{~cm}$, aspecto eunucoide (pubissuclo $86 \mathrm{W/2}$, envergadura 176). Vello axilar inexistente, escaso vello perigenital sin vello pubiano, mamas bien desarrolladas, aréolas amplias no pigmentadas, pezones pequeños. Cicatriz de herniorrafia inguinal bilateral. Manos y pies grandes.

Genitales externos femeninos, normales; clítoris, normal. Examen ginecológico: vagina larga que termina en fondo de saco. Mucosa vaginal rosada eutrófica. No se visualiza cuello uterino, no se palpa cuerpo uterino, ni anexos.

\section{Fxámenes:}

Cromatina nuclear, ( $\cdots$ ). Colpocitograma: deficiencia estrogénica mínima a moderada. GT urinarias: $18 \mathrm{U}$ st. $17 \mathrm{KS}$ : 12,1 mgs. Dermatoglifos: conteo 82; ángulo a t d: $42^{\circ}$; criptas anchas; manos grandes. Cariograma: $46 \mathrm{XY}$.

\section{CASO $\mathrm{N}^{+0} 4$}

I.B. 30 años, casada. Molestias principales: amenorrea y esterilidad. Por negarse a cooperar con el estudio sólo se tiene un cromatina nuclear $(-)$.

\section{COMENTARIO Y DISCUSION}

Existe acuerdo en considerar el síndrome de feminización total o testículo feminizante, como un cuadro de tipo hereditario para el que se postulan dos hipótesis respecto a su modo de transmisión: a) existencia de un gen recesivo ligado al sexo, o b) de un gen autosómico limitado al sexo y mutante.

En cualquiera de los dos casos el resultado sería un cariotipo de $46 \mathrm{XY}$, en que el gen anormal condicionaría alteraciones en la histología de las gónadas, en la síntesis hormonal y en la capacidad de respuesta de los efectores a las substancias hormonales, dando como resultante un individuo cario-típicamente masculino y fenotípicamente femenino. ${ }^{1-2-3-9}$

El estudio genético practicado en familias con individuos portadores de este síndrome, ha demostrado que la frecuencia de su presentación es de 63 hombres enfermos, por 20 hombres normales y por 75 mujeres aparentemente normales, entre las cuales hay un cierto número de portadores del alelo anormal. El análisis de los árboles genealógicos sugiere que la mujer portadora es heterocigota para un alelo anormal el que al ser recibido por el hombre (XY) de lugar a un pseudohermafroditismo masculino. ${ }^{11}$

Respecto a los dermatoglifos no hemos encontrado antecedentes en la literatura sobre sus características en este tipo de pacientes, pero en nuestros casos nos ha llamado la atención la presencia de papilas anchas y criptas profundas, características del sexo masculino. Este hallazgo no coincide con el conteo de las líneas, que ha sido bajo en todos nuestros casos, acercándose así al tipo femenino.

El ángulo ATD está dentro de límites normales.

La histología de las gónadas, que morfológicamente corresponden a testículos, está caracterizada por ausencia de las células germinales e hiperplasia de las células de Leyding, aspectos inmaduros de los túbulos seminíferos con engrosamiento de la membrana basal y cierto grado de fibrosis tubular a intersticial. ${ }^{1-5-6-7-8}$

Respecto al análisis de las determinaciones hormonales en lo que se refiere a los andrógenos plasmáticos, hay una proporción mayor de DHA en relación a la testosterona, siendo los valores de esta última normales para el hombre adulto y algo elevados para la mujer. Este hallazgo es paralelo al de la eliminación de los $17 \mathrm{KS}$ urinarios, que también están en cifras normales para el hombre y en valores normales altos o algo elevados para la mujer.

Se encuentran además pequeñas cantidades de pregnandiol y pregnanetriol urinarios. 
En lo que respecta a los estrógenos plasmáticos, sus niveles son normales bajos para la mujer y elevados para el hombre.

La excreción de $17 \mathrm{OH}$ urinarios es normal.

La GT urinarias y medición de LH están en valores normales para el hombre adulto. ${ }^{1-6-\theta}$

El test de frenación suprarrenal con Dexametasona permite concluir que una buena proporción de los $17 \mathrm{KS}$ urinarios, son de origen testicular, ya que se mantiene una frenación total de los $17 \mathrm{OH}$ en comparación con una disminución del 60 al $30 \%$ de los $17 \mathrm{KS}$, con sólo una leve caída de la androsterona y testoserona plasmática. Se aprecia un descenso a la mitad del pregnanetriol. ${ }^{6-9}$

Al efectuar el test de estimulación con FSH, no se observa un aumento significativo de los 17 KS urinarios, que sí se aprecia al estimular con HCG (gonadotrofinas coriónica humana) notándose especialmente un aumento de la androsterona, etiocolanolona y prenanetriol ${ }^{9}$.

Los estudios efectuados en los pacientes sometidos a gonadectomía, muestran una caída de la testosterona y estrona plasmáticas, 17 KS urinarios y pregnanetriol, coincidiendo con elevación de la GT urinarias a niveles semejantes a los de las mujeres postmenopáusicas. $^{6-1}$
A su vez, el estudio in vitro mediante la incubación del tejido testicular con substancias marcadas (progesterona, acetato, testosterona, etc.) con el objeto de tratar de determinar la existencia o no de un trastorno enzimático en la sintesis hormonal y su localización, no han permitido llegar a explicar claramente su patogenia.

Para algunos autores ${ }^{6.95}$ existiría una falla enzimática en el metabolismo de la testosterona, localizada a nivel de la 3 kol dehidrogenasa; ello determinaría que en la síntesis de testosterona, se emplea especialmente la vía de la $\Delta 4$ progesterona en lugar de la $\Delta 5$ pregnenolona, que es la más usada normalmente. Otros autores creen que se produciría una elevada proporción de estrógenos en el testículo $^{9}$ y más específicamente, a nivel de las células de Leydig, ya que la transformación periférica de testosterona a estrógenos ha sido descartada. ${ }^{1}$

Por otra parte, el fracaso en obtener masculinización con grandes dosis de testosterona, aun después de la castración, hacen indispensable postular la existencia de un defecto genético de los órganos efectores, que los haría refractarios a los andrógenos. ${ }^{10-69-1}$

SINDROME DE FEMINIZACION TOTAL - CLINICA

\begin{tabular}{|c|c|c|c|c|c|c|c|}
\hline Nombre & $\begin{array}{l}\text { Edad } \\
\text { (años) }\end{array}$ & Talla & $\begin{array}{l}\text { Motivo } \\
\text { consulta } \\
\end{array}$ & $\begin{array}{l}\text { Mamas-desart. } \\
\text { V. axilar pigm. }\end{array}$ & $\begin{array}{l}\text { upu- } \\
\text { biano }\end{array}$ & Ex. Ginecológico & Ant. Imp. \\
\hline M.C. & 14 & $\begin{array}{l}167,5 \mathrm{~cm} \\
\text { Pub-suelo } \\
88 \\
\text { Enverg. } \\
178\end{array}$ & $\begin{array}{l}\text { Amenorrea } \\
\text { primaria. }\end{array}$ & $(+)( \pm)(-)$ & $\begin{array}{l}\text { Escaso } \\
\text { Peri- } \\
\text { vulvar. }\end{array}$ & $\begin{array}{l}\text { Clítoris pequeño. } \\
\text { Vagina corta: } 3 \text { o } 4 \mathrm{~cm} \text {. ter- } \\
\text { mina en forma ciega. } \\
\text { No se palpan útero ni } \\
\text { anexos. }\end{array}$ & $\begin{array}{l}\text { Op. } \\
\text { Hernia } \\
\text { Hernia } \\
\text { inguinal } \\
\text { bilateral. }\end{array}$ \\
\hline O.B. & 37 & $\begin{array}{l}160,5 \mathrm{~cm} \\
\text { Pub-suelo } \\
84 \\
\text { Enverg. } \\
165\end{array}$ & $\begin{array}{l}\text { Esterilidad. } \\
\text { Amenorrea } \\
\text { primaria. }\end{array}$ & $(+++)( \pm)( \pm)(-)$ & $\begin{array}{l}\text { Escaso } \\
\text { Peri- } \\
\text { vulvar }\end{array}$ & $\begin{array}{l}\text { Clítoris pequeño. } \\
\text { Labios mayores y meno- } \\
\text { res normales. Vagina lar- } \\
\text { ga term. en fondo de saco. } \\
\text { Utero y anexos }(-) \text {. }\end{array}$ & \\
\hline C.A. & 24 & $\begin{array}{l}167 \mathrm{~cm} \\
\text { Pub-suelo } \\
86.5 \\
\text { Enverg. } \\
176\end{array}$ & $\begin{array}{l}\text { Amenorrea } \\
\text { primaria. }\end{array}$ & $(+++)( \pm)(-)(-)$ & $\begin{array}{l}\text { Escaso } \\
\text { Peri- } \\
\text { vulvar }\end{array}$ & $\begin{array}{l}\text { Clítoris normal. Labios } \\
\text { mayores y menores norma- } \\
\text { les. Vagina larga term. } \\
\text { en fondo de saco. No hay cue- } \\
\text { llo, cuerpo, ni anexos. }\end{array}$ & $\begin{array}{l}\text { Op. } \\
\text { Hernia } \\
\text { inguinal } \\
\text { bilateral. }\end{array}$ \\
\hline 1.B. & 30 & & $\begin{array}{l}\text { Esterilidad } \\
\text { Amenorrea } \\
\text { primaria. }\end{array}$ & . & & & \\
\hline
\end{tabular}




\begin{tabular}{|c|c|c|c|c|c|c|c|c|}
\hline Nombre & $\begin{array}{l}\text { Edad } \\
\text { (años) }\end{array}$ & $\begin{array}{c}\text { Croma- } \\
\text { tina }\end{array}$ & $\begin{array}{l}\text { Colpact- } \\
\text { tograma }\end{array}$ & $G T$ & $\begin{array}{c}17 K s \\
y O H S\end{array}$ & $\begin{array}{c}\text { Caplac. } \\
\text { yodo }\end{array}$ & Glifos & $\begin{array}{l}\text { Cario- } \\
\text { grama }\end{array}$ \\
\hline M.C & 14 & $(-)$ & $\begin{array}{l}\text { Efecto } \\
\text { Estrogénico } \\
\text { normal. }\end{array}$ & 17 ust. & & 20 & $\begin{array}{l}\text { Manos grandes. } \\
\text { Papilas altas y gruesas. } \\
\text { Conteo total } 96 \\
\text { Angulo atd. } 44^{\circ} \text {. }\end{array}$ & $46 \mathrm{XY}$ \\
\hline O.B & 37 & $(-)$ & Demod. & 10 ust & & & $\begin{array}{l}\text { Manos grandes. } \\
\text { Conteo total } 110 \\
\text { Angulo atd. } 47^{\circ}\end{array}$ & $46 X Y$ \\
\hline C.A. & 24 & $(-)$ & $\begin{array}{l}\text { Demin. a } \\
\text { Demod. }\end{array}$ & 18 ust. & $\begin{array}{l}12,1 \mathrm{mg} . \\
12,9 \mathrm{mg} .\end{array}$ & & $\begin{array}{l}\text { Manos grandes. } \\
\text { Criptas anchas. } \\
\text { Conteo total } 82 \\
\text { Angulo atd. } 42^{\circ}\end{array}$ & $46 X Y$ \\
\hline
\end{tabular}

I.B. $30 \quad(-)$

Existen trabajos recientes que apoyan estas hipótesis postulando la existencia de un bloqueo en la transformación periférica de testosterona a 5 a dehidrotestosterona que sería el derivado activo. En todo caso, la investigación sigue abierta y aún no se ha establecido con certeza la patogenia del cuadro. ${ }^{10}$

\section{TRATAMIENTO}

La gónada de estos individuos es necesaria y suficiente para inducir un crecimiento normal y un desarrollo puberal femenino satisfactorio, aunque incompleto y acompañado de amenorrea y esterilidad, de tal manera que hasta que no se haya completado este desarrollo, no será necesario indicar tratamiento alguno.

El conflicto se presenta una vez pasados los 20 años, respecto al criterio conductual con estas gónadas defectuosas.

Se ha aducido que la extirpación de estos testículos traería una defeminización con síntomas subjetivos que recuerdan la menopausia femenina normal, lo que podría afectar la conducta sexual de estas "mujeres" que tienen atracción por el sexo masculino y cuyas relaciones son completamente normales y con orgasmo. La verdad es que estos trastornos no siempre se producen y cuando ello sucede se pueden controlar perfectamente con terapia estrogénica substitutiva.
Por otro lado, se sabe que estos testículos tienen una gran tendencia a la malignización, Morris y Maheesch ${ }^{12}$ anotan un $22 \%$ en una serie y Calatroni-Ruiz ${ }^{13}$ relatan una serie de 28 casos en los cuales hubo 7 casos de malignización (25\%) contra un caso (5\%) que se encuentra en otros de pseudohermafroditismo masculino con testículos también ectópicos.

Este motivo inclina a la mayoría de los autores ${ }^{14-15-16-17}$ a efectuar la castración una vez obtenido el desarrollo puberal completo.

Tan importante como esto es el tacto que debe tener el médico tratante para guardar el secreto profesional frente a la paciente y sus familiares, quienes en ningún momento deben sospechar el trastorno, lo que de una u otra forma traería como consecuencia profundas alteraciones de la futura vida sexual de la niña. Tan sólo se los pondrá en antecedentes respecto a la amenorrea y esterilidad definitivas.

\section{RESUMEN}

- Se presentan cuatro casos familiares de síndrome de feminización total.

- Se realiza estudio clínico-genético y de laboratorio.

- Se discute la patogenia del cuadro, su forma de transmisión y su tratamiento.

\section{SUMMARY}

Four related patients with Testicular feminization syndrome are presented. They are $14-24-30$ and 37 years old

Their main complaine vere primary amenorrhea and 
sterillity. They are fenotipically femenine but they do not have uterus and anexo. The show ennucoid proportions with big hands and feet. They do not have sexual hair. Their cariogram showed an XY chromosome pattern.

The theories explaining the ethiopathogenia and way of Iransmision of this syndrome are analised and the psycological aspects of it are discussed.

\section{REFERENCIAS}

Williams, R.H. Tex Book of Endocrinology, 1968.

Hubble, D. Paediatric Endocrinology, 1969.

Tohm Dozumi. Acte Endocr., 54:197, 1967.

Jacob. Lancet II 591, 1959.

Chaurreau, E. J.C. Endo, Vol. 28/XII/68.

- Frech, F.S. J C1. Endo, 25:661-1965 y 1966
7 Griffithe. J. Cl. Endo, 23:1044, 1963.

8 Ruce, B.F.J.CI. Endo, 27:29, 1967.

- Wiener, S. J. Cl. Endo, 25:1393, 1965.

10 Northout, R. J.Cl. Endo, 29:417, 1969.

11 Stem. C. Principles of Human Genetic, 1960

12 Mirni, Mahie'sh Am. J Obst. Gyner, 67:731, 1963.

13 Galatroni, C. y $V$ Ruzz. Terapéutica ginecológica. Ed. El Ateneo, Buenos Aires, 1970, pág. 201.

14 Jones, H.W. and W.N. Scott. Hermafroditism, Genital Abnormalities and related Endocrine disorders. Ed. The Williams and Wilkins Co., Baltimore, 1958.

15 Botella Llusiá, J. Endocrinología de la mujer. Ed. Científico-Médica, Barcelona, 1961.

16 Jeffenste, $N$ Ginecología. Ed. Intermédica, Buenos Aires, 1971.

17 Van $W_{y c k} J . J$. The pathogenesis of Sexual Anomalies. The Ovary. Ed. Grady, H.C. and Smith D.E. The Williams and Wilkins Co., Baltimore, 1963, pág. 41.

Trabajo entregado para su publicación en 1975. 